УДК 665.63: 51.001.57

\title{
МНОГОКРИТЕРИАЛЬНАЯ ОПТИМИЗАЦИЯ ПРИ УПРАВЛЕНИИ ХИМИКО-ТЕХНОЛОГИЧЕСКОЙ СИСТЕМОЙ ПРОИЗВОДСТВА БЕНЗОЛА ПРИ НЕЧЕТКОЙ ИНФОРМАЦИИ
}

\author{
Оразбаев Батыр Бидайбекович', \\ batyr_o@mail.ru \\ Оспанов Ербол Амангазович', \\ 780ea@mail.ru \\ Оразбаева Кульман Нахановна², \\ kulman_o@mail.ru \\ Серимбетов Булат Абуталибович², \\ sba_mmc@mail.ru \\ ' Евразийский национальный университет им. Л.Н. Гумилева, \\ Казахстан, 010008, г. Астана, ул. Пушкина, 11. \\ 2 Казахский университет экономики, финансов и международной торговли, \\ Казахстан, г. Астана, ул. Жубанова, 7.
}

\begin{abstract}
Актуальность исследования обусловлена необходимостью эффективного решения задач управления режимами работы химико-технологических систем, которые являются многокритериальными, характеризуются неопределенностью и нечеткостью исходной информации. Так как реальные химико-технологические системы на производстве являются многокритериальными и часто работают в условиях нечеткости исходной информации, формализация и эффективное решение таких задач в нечеткой среде является весьма важной и актуальной для науки и производства.

Цель: разработать метод построения математических моделей химико-технологических систем на основе доступной информации различного характера (теоретической, статистической, экспертной, нечеткой информации); получить постановку задачи многокритериальной оптимизации при управлении такими системами и разработать эвристический подход кее решению. Также провести апробацию полученных результатов на практике для построения математических моделей химико-технологической системы производства бензола и управления режимами ее работы в нечеткой среде.

объекты: сложные химико-технологические системы на примере технологической установки производства бензола, которые описываются вектором критериев и характеризуются нечеткостью исходной информации.

Методы: методы системного анализа и теорий нечетких множеств, методы математического моделирования и многокритериального выбора, экспериментально-статистические методы разработки математических моделей.

Результаты. Предложен метод разработки математических моделей химико-технологических систем на основе доступной информации различного характера, в том числе исходной нечеткой информации. Новые постановки задач управления химикотехнологическими системами в условиях нечеткости исходной информации получены путем модификации различных схем компромиссов для работы в нечеткой среде. Задачи многокритериальной оптимизации при управлении технологической системой сведены к многокритериальным задачам нечеткого математического программирования и на основе модификации принципов максимина и Парето оптимальности разработан эвристический алгоритм решения сформулированной задачи оптимального управления. Эти результаты реализованы на практике при построении математических моделей основных агрегатов химикотехнологической системы производства бензола и при постановке и решении задачи управления режимами работы этой системы в нечеткой среде. Анализ и сравнение полученных результатов позволяют сделать вывод об эффективности предложенного подхода к решению задач управления на основе моделей в условиях нечеткости исходной информации.
\end{abstract}

\section{Ключевые слова:}

Химико-технологическая система, математическое моделирование, нечеткая информация,

принципы оптимальности, бензол, эвристический алгоритм, задачи управление режимами работы.

\section{Введение}

Одним из перспективных и эффективных подходов к оптимизации и управлению режимами работы сложных химико-технологических систем (ХTC) в условиях неопределенности, вызванной нечеткостью исходной информации, является подход, использующий экспертные методы [1-4] и методы нечетких множеств [5-10]. Технологические объекты нефтеперерабатывающих производств относятся к сложным ХTC, в которых протекают технологические процессы по переработке сырья и производства нефтепродуктов, часто функционируют при нечеткой информации. Поэтому для мно- гокритериальной оптимизации при управлении режимами работы таких систем необходимо учесть вектора критериев и нечеткость исходной информации. Такие задачи, как правило, формализуются в виде многокритериальных задач, которые решаются на основе знаний и опыта специалистов-экспертов, лица, принимающего решения (ЛПР), и математических моделей объектов и протекающих в них процессов, построенных с учетом нечеткости исходной информации $[11,12]$. Такой подход привел к появлению методов решения задач многокритериальной оптимизации при управлении системой в нечеткой среде, использующие методологию 
системного анализа, знания и опыт ЛПР, его предпочтения при выборе решений [13-16].

С целью формализации и применения исходной нечеткой информации для построения математических моделей и управления ХTC можно успешно использовать методы экспертных оценок и теории нечетких множеств (ТНM). Для успешного решения проблем моделирования и оптимизации при управлении режимами работы, векторной оптимизации параметров ХTC в условиях неопределенности, нечеткости необходимо разработать метод построения моделей на основе доступной информации различного характера, дальше развивать методы решения задач многокритериальной оптимизации при управлении режимами работы ХTC в нечеткой среде, которые являются актуальными задачами химической технологии, нефтепереработки и нефтехимии [17].

В качестве объекта исследования рассматривается XTC производства бензола Атырауского нефтеперерабатывающего завода (НПЗ), для которой необходимо определить оптимальные значения режимных параметров, обеспечивающие эффектив- ное управление режимами работы по вектору критериев при ограничениях, имеющих нечеткий характер. При этом основными задачами являются: разработка математических моделей основных агрегатов объекта исследования, постановка задачи многокритериальной оптимизации при управлении XTC производства бензола в условиях неопределенности и разработка эвристического алгоритма ее решения [18].

Рассмотрим основные характеристики XТC производства бензола как объекта управления. На рисунке приведена схема объекта управления и процесса управления ХTC производства бензола.

Входными и режимными параметрами объекта управления, которые влияют на его режимы работы, являются: объем сырья, т. е. реформата $\left(x_{1}\right)$, и его качественные показатели: доля серы $\left(x_{4}\right)$ и ароматических углеводородов (УВ) в составе сырья $\left(x_{5}\right)$, а также температура $\left(x_{2}\right)$ и давление в бензольной колонне (БК) $\left(x_{3}\right)$. Эти параметры являются режимными параметрами, изменениями которых определяются оптимальные режимы работы ХТC производства бензола (рисунок).

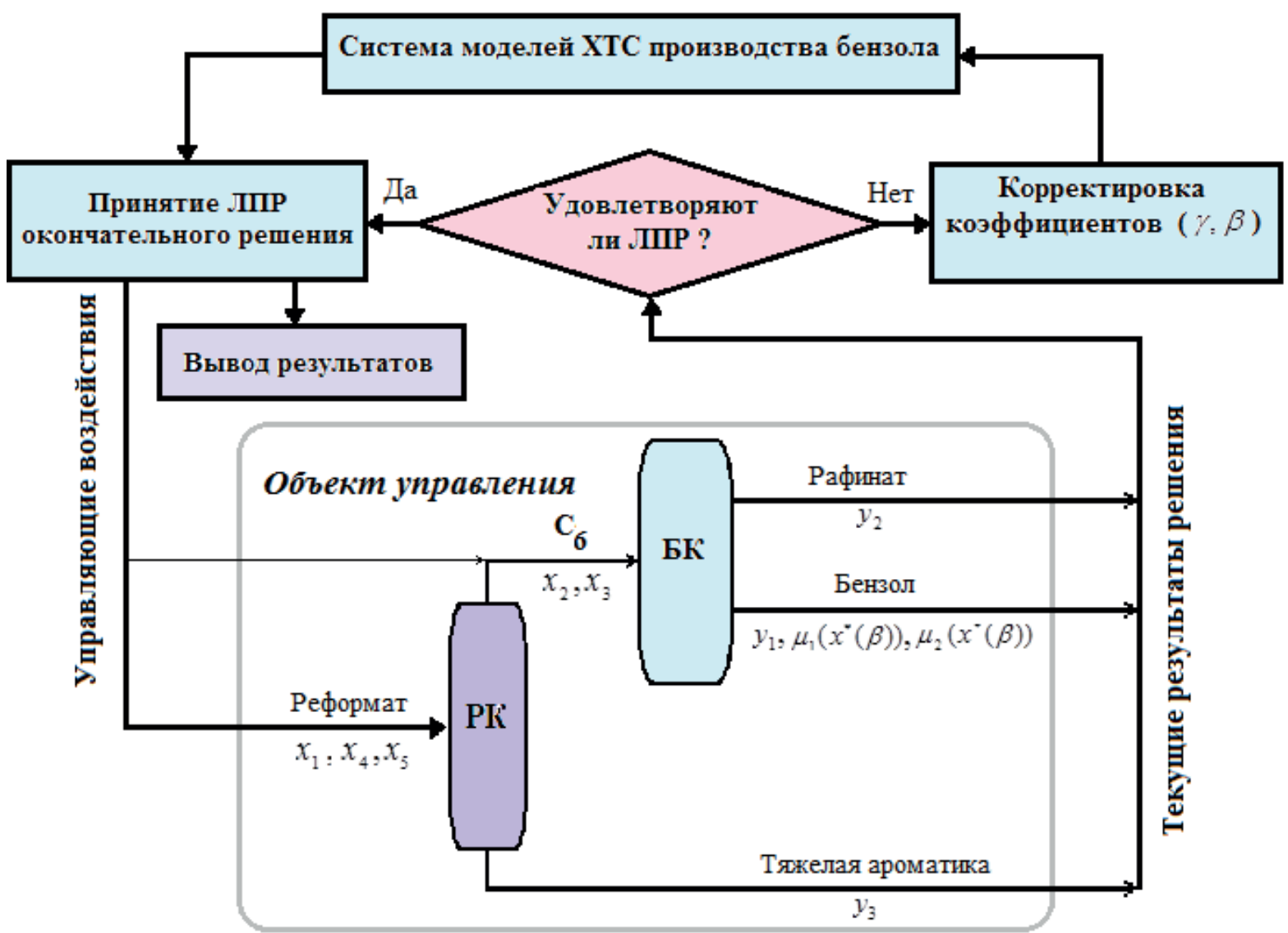

Рисунок. Схема объекта управления и процесса управления ХТС производства бензола: БК - бензельная колонна; РК - ректифика иионная колонна; $\gamma$ и $\beta$-вектор весовых коэффициентов локальных критериев и ограничений; $x_{1}, x_{2}, x_{3}, x_{4}, x_{5}-$ режимные па раметры, соответственно: сырье, вход реформата, $x_{1}$, тыс. т; температура в бензольной колонне, $x_{2}$ в K; давление в бензольной колонне, $x_{3}$, Па; доля серы в составе сырья, $x_{4}, \%$; доля ароматических углеводородов в составе сырья, $x_{5}$, \%

Figure. Scheme of the control object and the control process of the Chemical-technological system of benzene production: $5 K$ is the benzene column; $\gamma$ and $\beta$ are the vector of weight coefficients of local criteria and constraints; $x_{1}, x_{2}, x_{3}, x_{4}, x_{5}$ - regime parameters, respectively: raw materials, Reformat input, $x_{1}$, thousand tons; temperature in a benzole column, $x_{2}, K$; pressure in the benzene column, $x_{3}$, Pa; share of sulfur in the composition of raw materials, $x_{4}, \%$; share of aromatic hydrocarbons in the composition of raw materials, $x_{5}$, $\%$ 
Выходными параметрами объекта управления, т. е. критериями управления являются: выход целевой продукции - бензола $\left(y_{1}\right)$, объемы рафината $\left(y_{2}\right)$ и тяжелой ароматики $\left(y_{3}\right)$. При этом также необходимо контролировать качественные показатели бензола следующими нечеткими ограничениями: "среднее октановое число бензола не менее...»; «содержание серы в составе бензола не более...», выполнение которых обеспечивается путем контроля степени выполнения (функции принадлежности) этих нечетких ограничений: $\mu_{1}(x)$; $\mu_{2}(x)$. Процесс управления осуществляется в диалоговом режиме, на основе моделей и эвристического алгоритма с учетом предпочтения ЛПР. В результате решения задачи оптимизации режимов работы ХТС производства бензола определяются значения режимных параметров $\left(x_{1}, x_{2}, x_{3}, x_{4}, x_{5}\right)$, обеспечивающие лучшие значения критериев управления, не нарушая наложенных ограничений.

Конкретизируем основные параметры задачи многокритериальной оптимизации при управлении режимами работы ХТС производства бензола. Известен вектор критериев управления: $\mathbf{f}(x)=\left(f_{1}(x), f_{2}(x), f_{3}(x)\right)$, где $f_{1}(x)$ - выход бензола, $f_{2}(x)$ - выход рафината с бензольной колонны, $f_{3}(x)$ - выход тяжелой ароматики с ректификационной колонны (РК). Известны минимальные и максимальные значения рассматриваемых критериев: $f_{1}(x)=\left[(1,27 \ldots 1,38) \cdot 10^{8} \kappa \Gamma\right] ; f_{2}(x)=\left[(7,7 \ldots 8,6) \cdot 10^{7} \kappa \Gamma\right] ;$ $f_{3}(x)=\left[(4,45 \ldots 4,56) \cdot 10^{8}\right.$ кг $][19]$.

Вектор входных и режимных параметров $\mathbf{x}=\left(x_{1}, x_{2}, x_{3}, x_{4}, x_{5}\right)$ влияет на значения критериев $f_{1}(x), f_{2}(x), f_{3}(x)$ и нечетких ограничений $\varphi(x)$. Они могут измеряться с помощью соответствующих измерительных приборов $\left(x_{1}, x_{2}, x_{3}\right)$ или определяться путем лабораторного анализа с участием человека $\left(x_{4}, x_{5}\right)$. Интервалы изменения режимных параметров известны [20]. На основе методов экспертных оценок с привлечением ЛПР для ХТС производства бензола Атырауского НПЗ построены функции принадлежности (ФП) выполнения нечетких ограничений $\varphi(x)=\left(\varphi_{1}(x), \varphi_{2}(x)\right)-\mu_{1}(x)$ и $\mu_{2}(x)$, «среднее октановое число бензола должно быть не менее чем $(\tilde{\geq}) 102 »$, и «содержание серы в бензоле

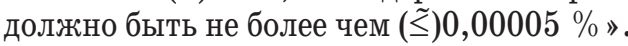

\section{Метод построения математических моделей XTC на основе информации различного характера, разработка моделей производства бензола в нечеткой среде}

Для математической формулировки и определения зависимости каждого частного критерия $f_{i}(x), i=\overline{1,3}$ от режимных параметров необходимо разработать математические модели основных агрегатов XTC [11, 21, 22], описывающие зависимости критериев управления системой от ее режимных параметров. На основе методологии системного анализа нами предлагается следующий метод построения моделей ХTС с использованием до- ступной информации различного характера, состоящий из следующих этапов (пунктов):

1) исследование элементов ХТС, связи между элементами, сбор и обработка доступной информации, определение цели моделирования;

2) определение критериев оценки, сравнения и выбора моделей, которые можно построить для элементов ХTC с учетом цели моделирования;

3) по выбранным критериям проведение экспертной оценки, т. е. ранжирования видов моделей, которые возможно построить для каждого элемента ХTС и по сумме значений рангов определить оптимальный тип модели каждого элемента системы [11];

3.1) если теоретические сведения для описания работы элемента ХТС достаточны и по сумме значений критериев, т. е. рангов оценки, построение детерминированной модели является эффективным, то для этого элемента на основе аналитического метода строится детерлинированная модель;

3.2) если статистические данные для описания работы элемента ХTC доступны и по сумме рангов статистическая модель набирает наилучшее значение, то статистические модели этого элемента строятся на основе экспериментально-статистических методов;

3.3) если теоретические и статистические данные для описания элемента ХTC недостаточны и сбор таких данных экономически нецелесообразен, а нечеткая информация, описывающая работу данного элемента, доступна и по сумме оценки рангов нечеткая модель набирает наибольший ранг, то для него на основе методов ТНМ строятся нечеткие модели в соответствии с пунктом 4 ;

3.4) если теоретическая, статистическая и нечеткая информация для описания работы элемента ХTC недостаточна и построение детерминированных, статистических и нечетких моделей для этого объекта сложно или невозможно, нецелесообразно, то для этого элемента путем комбинирования доступной информации различного типа (теоретическая, статистическая, нечеткая) строится комбинированная модель. Для этого, в зависимости от характера исходной информации, перейти к пунктам 3.1-3.3 или 4;

4) определяются необходимые для построения модели нечеткие входные, режимные $\tilde{x}_{i} \in \tilde{A}_{i}, i=\overline{1, n}$ и выходные (критерии) $\tilde{y}_{j} \in \tilde{B}_{j}, j=\overline{1, m}$ параметры, где $\tilde{A}_{i} \in X, \tilde{B}_{j} \in Y$ - нечеткие подмножества, принадлежащие универсальным множествам $X, Y$. Причем параметры $x_{i} \in X_{i}, i=\overline{1, n}$ могут быть четкими;

5) если входные параметры объекта четкие, т. е. $x_{i} \in X_{i}, i=\overline{1, n}$, то определяются структуры нечетких уравнений множественной регрессии $\tilde{y}_{j}=f_{i}\left(x_{1}, \ldots, x_{n}, \tilde{a}_{0}, \tilde{a}_{1}, \ldots \tilde{a}_{n}\right), j=\overline{1, m}$ (структурная идентификация), например, на основе метода последовательного включения регрессоров; 
6) на основе методов экспертных оценок с привлечением ЛПР описывается объект (элемент ХТС) и определяется терм-множество нечетких параметров $T\left(X_{i}, Y_{j}\right)$;

7) построение $Ф П$ нечетких параметров $\mu_{A_{i}}\left(\tilde{x}_{i}\right)$, $\mu_{B_{i}}\left(\tilde{x}_{j}\right)$. Для построения ФП, например, выходных параметров объекта, предлагается использовать следующую формулу:

$$
\mu_{B_{j}}^{p}\left(\tilde{y}_{j}\right)=\exp \left(Q_{B_{j}}^{p}\left|\left(y_{j}-y_{m d j}\right)^{N_{B_{j}}^{p}}\right|\right) .
$$

Здесь и далее $p$ - номер кванта; $Q_{B_{i}}^{p}$ параметр, характеризующий степень нечеткости, его значение идентифицируется при построении $Ф П ; N_{B_{2}}^{p}-$ коэффициент, позволяющий более точно аппроксимировать график $Ф П ; y_{m d j}^{p}$ - такая нечеткая переменная, которая более точно соответствует выбранному терму и определяется выражением

$$
\mu_{B_{j}}^{p}\left(y_{m d i}\right)=\max _{j} \mu_{B_{j}}^{p}\left(y_{j}\right) ;
$$

8) если режимные параметры и критерии, т. е. входные и выходные параметры элемента ХTC, являются нечеткими (лингвистические переменные), то формализуются нечеткие отображения $R_{i j}$, которые определяют связи между этими лингвистическими переменными $\tilde{x}_{i}$ и $\tilde{y}_{j}$. Строятся лингвистические модели с переходом к пункту 10 ;

9) если условие пункта 5 выполняется, т. е. входные, режимные параметры четкие, то оцениваются значения нечетких коэффициентов $\left(\tilde{a}_{0}, \tilde{a}_{1}, \ldots, \tilde{a}_{n}\right)$ моделей $\tilde{y}_{j}$ (параметрическая идентификация), структура которых идентифицирована в пункте 5 и осуществляется переходом к пункту 11 ;

10)если условие пункта 8 выполняется, т. е. и входные и выходные параметры элемента ХTC нечеткие, то на основе правил композиционного вывода $B_{j}=A_{j} R_{i j}$ определяются нечеткие значения выходных параметров объекта:

$$
\mu_{B_{j}}^{p}\left(\tilde{y}_{j}^{*}\right)=\max _{x_{i} \in X_{i}}\left\{\min \left[\mu_{A_{i}}^{p}\left(\tilde{x}_{i}^{*}\right), \mu_{R_{i j}}^{p}\left(\left(\tilde{x}_{i}^{*}, \tilde{y}_{j}\right)\right]\right\},\right.
$$

где $\mu_{B_{i}}^{p}\left(\tilde{y}_{j}^{*}\right)$ - функция принадлежности нечетких выходных параметров на $p$-м кванте. Четкие, т. е. числовые значения выходных параметров объекта $\tilde{y}_{j}^{* * *}$ определяются из множества нечетких решений (2) по формуле:

$$
\tilde{y}_{j}^{* *}=\arg \max _{\tilde{y}_{j}^{*}} \mu_{B_{j}}^{*}\left(\tilde{y}_{j}^{*}\right) \text {; }
$$

11)проверяется адекватность модели. Если условие адекватности выполняется: т. е. $S=\left|y_{m}-y_{e}\right| \leq S_{D}$, где $S$ и $S_{D}-$ значение критерия для оценки адекватности и его допустимое значение; $y_{m}$ и $y_{e}-$ значения выходных параметров, полученных по модели $\left(y_{m}\right)$ и по результатам экспериментов $\left(y_{e}\right)$, соответственно, при одинаковых значениях входных параметров, то разработанные модели считаются адекватными и рекомендуются для применения. В противном случае, выясняется причина неадекватности и осуществляется возврат к соответствующим пунктам для устранения причин неадекватности и достижения адекватности.
Используя предложенный метод, модели ХТС производства бензола определены в виде нелинейных регрессионных уравнений, которые описывают зависимость частных критериев (выходы бензола, рафината и тяжелой ароматики) от режимных параметров $x_{1}, x_{2}, x_{3}$ :

$$
\begin{gathered}
f_{1}\left(x_{1}, x_{2}, x_{3}\right)= \\
=0,099849 x_{1}+0,020462 x_{2}-0,76 x_{3}+ \\
+0,000148 x_{1}^{2}+0,000008 x_{2}^{2}-0,032571 x_{3}^{2}+ \\
+0,000046 x_{1} x_{2}+0,000571 x_{1} x_{2}-0,000585 x_{2} x_{3}+ \\
+0,000571 x_{1} x_{3}-0,000585 x_{2} x_{3} ; \\
f_{2}\left(x_{1}, x_{2}, x_{3}\right)=0,061562 x_{1}+0,012615 x_{2}- \\
-0,234286 x_{3}+0,000074 x_{1}^{2}-0,000015 x_{2}^{2}+ \\
+0,013388 x_{3}^{2}+0,000009 x_{1} x_{2}+ \\
+0,001055 x_{1} x_{3}-0,000180 x_{2} x_{3} ; \\
f_{3}\left(x_{1}, x_{2}, x_{3}\right)=0,000000001+0,0418920 x_{1}- \\
-0,171690 x_{2}+3,188570 x_{3}+0,000630 x_{1}^{2}- \\
-0,00013 x_{2}^{2}+0,136653061 x_{3}^{2}+0,00006 x_{1} x_{2}+ \\
+0,00718 x_{1} x_{3}-0,00123 x_{2} x_{3} .
\end{gathered}
$$

Параметры уравнений (3)-(5) определены с помощью метода наименьших квадратов (с применением программ множественной регрессии «Regress»). На значения $f_{1}(x), f_{2}(x), f_{3}(x)$ параметры $x_{4}-$ доля серы, и $x_{5}$ - доля ароматических углеводородов (УВ) в составе сырья, оказывают очень слабое влияние, поэтому ими можно пренебречь.

Для математической формализации нечетких ограничений $\varphi_{1}(x), \varphi_{2}(x)$ использованы методы THM, с представлением их в виде нечетких уравнений регрессии. После параметрической идентификации коэффициентов уравнений нечетких регрессий с применением системы MatLab [23] и преобразования на основе множеств уровня $\alpha$ (для уровней $\alpha=0,5 ; 0,8 ; 1)$ идентифицированы нечеткие ограничения $\varphi_{1}\left(x_{2}, \ldots, x_{5}\right) \tilde{\geq} 102$ и $\varphi_{2}\left(x_{2}, \ldots, x_{5}\right) \tilde{\leq} 0,00005$.

Например, нечеткое ограничение $\varphi_{1}(x)$ - «среднее октановое число бензола $\tilde{\geq} 102$ будет иметь вид:

$$
\begin{aligned}
& \varphi_{1}\left(x_{2}, \ldots, x_{5}\right)= \\
& =\left(\frac{0,5}{0,0231}+\frac{0,8}{0,0233}+\frac{1}{0,0235}+\frac{0,8}{0,0237}+\frac{0,5}{0,0239}\right) x_{2}- \\
& -\left(\frac{0,5}{0,5823}+\frac{0,8}{0,5825}+\frac{1}{0,5828}+\frac{0,8}{0,58}+\frac{0,5}{0,5834}\right) x_{3}- \\
& +\left(\frac{0,5}{5000}+\frac{0,8}{5050}+\frac{1}{5100}+\frac{0,8}{5150}+\frac{0,5}{520}\right) x_{4}+ \\
& +\left(\frac{0,5}{1,01}+\frac{0,8}{1,015}+\frac{1}{1,02}+\frac{0,8}{1,025}+\frac{0,5}{1,03}\right) x_{5}+ \\
& +\left(\begin{array}{l}
\frac{0,5}{0,000014}+\frac{0,8}{0,000016}+\frac{1}{0,000018}+ \\
+\frac{0,8}{0,00002}+\frac{0,5}{0,000022}
\end{array}\right) x_{2}^{2}-
\end{aligned}
$$




$$
\begin{aligned}
& -\left(\begin{array}{l}
\frac{0,5}{0,01655}+\frac{0,8}{0,0166}+\frac{1}{0,01665}+ \\
+\frac{0,8}{0,0167}+\frac{0,5}{0,01675}
\end{array}\right) x_{3}^{2}- \\
& -\left(\frac{0,5}{12747}+\frac{0,8}{12748,5}+\frac{1}{1275}+\frac{0,8}{12751,5}+\frac{0,5}{12753}\right) x_{4}^{2}+ \\
& +\left(\begin{array}{l}
\frac{0,5}{0,02443}+\frac{0,8}{0,02446}+\frac{1}{0,02448}+ \\
+\frac{0,8}{0,02450}+\frac{0,5}{0,024530}
\end{array}\right) x_{5}^{2}+ \\
& +\left(\begin{array}{l}
\frac{0,5}{0,00042}+\frac{0,8}{0,000450}+\frac{1}{0,000470}+ \\
+\frac{0,8}{0,00049}+\frac{0,5}{0,00052}
\end{array}\right) x_{2} x_{5}- \\
& -\left(\begin{array}{l}
\frac{0,5}{145,680}+\frac{0,8}{145,7040}+\frac{1}{145,7140}+ \\
+\frac{0,8}{145,7240}+\frac{0,5}{145,740}
\end{array}\right) x_{3} x_{4}- \\
& -\left(\begin{array}{l}
\frac{0,5}{0,00578}+\frac{0,8}{0,00580}+\frac{1}{0,00581}+ \\
+\frac{0,8}{0,00582}-\frac{0,5}{0,00583}
\end{array}\right) x_{3} x_{5}+ \\
& +\left(\frac{0,5}{48,00}+\frac{0,8}{50,00}+\frac{1}{51,00}+\frac{0,8}{52,00}+\frac{0,5}{54,00}\right) x_{4} x_{5} \tilde{\geq} 102 .
\end{aligned}
$$

Аналогично представляется нечеткое ограничение «содержание серы в бензоле должно быть не более чем $(\tilde{\leq}) 0,00005 \%$ », т. е. $\varphi_{2}\left(x_{2}, \ldots, x_{5}\right) \tilde{\check{\Sigma}} 0,00005$.

Так как функции принадлежности нечетких коэффициентов, как правило, имеют колокообразный вид (1), имеются левые 0,5 и 0,8 , а также правые 0,8 и 0,5 значения $\alpha$-уровней. Для более удобного расчета производится процесс дефаззификации [23].

Для определения влияния ароматических углеводородов в составе реформата на среднее октановое число бензола на основе предложенного метода построены лингвистические модели, которые реализуют логический вывод «Чем больще ароматических углеводородов в составе сырья, тем больше среднее октановое число бензола»:

$$
\begin{aligned}
& \text { if } \tilde{x} \in \tilde{A}_{1} \text {, then } \tilde{y} \in \tilde{B}_{1} \text {, if } \tilde{x} \in \tilde{A}_{2} \text {, then } \tilde{y} \in \tilde{B}_{2} \text {, } \\
& \text { else if } \tilde{x} \in \tilde{A}_{3} \text {, then } \tilde{y} \in \tilde{B}_{3} \text {, }
\end{aligned}
$$

где $A_{1}=n z, B_{1}=n n, A_{2}=n r, B_{2}=n r, A_{3}=v s, B_{3}=v n$,; $\tilde{A}_{\text {i }}$, $\tilde{B}_{j}, i=1,3 ; j=1,3-$ нечеткие подмножества $(n z-$ «низкое», $s r-$ «ниже нормы», $s r-$ «среднее», $n r-$ «нормальное», vs - «высокое», vn - «выше нормы» лингвистические значения); $\tilde{x}$ и $\tilde{y}$ - входные и выходные лингвистические переменные, описывающие режимные параметры и критерии, которые оценивают качество сырья и среднее октановое число бензола. Модели отображают связь между $\tilde{x}$ и $\tilde{y}$.

Для многокритериальной оптимизации при управлении ХTC производства бензола необходимо определить и выбрать такие значения режимных параметров $x_{1}, x_{2}, x_{3}, x_{4}, x_{5}$, являющиеся управляющими воздействиями, которые обеспечивают выбранным критериям $f_{i}(x), i=1, m$ экстремальные значения. При этом необходимо обеспечить выполнение ограничений, соблюдение технологических регламентов, которые позволяют вести процесс в устойчивом и безаварийном режиме $(x \in X$; $\left.X=\left\{x \in \Omega, \varphi_{q}(x) \tilde{s}_{q}, q=1,2\right\}, x=\left[x^{\min }, x^{\max }\right]\right)[24]$.

\section{Задача многокритериальной оптимизации при управлении режимами работы XTC в нечеткой информационной среде \\ и эвристический метод ее решения. Результаты}

Так как в сформулированной нами задаче многокритериальной оптимизации при управлении режимами работы XТС производства бензола ограничения $\varphi_{1}(x)$ и $\varphi_{2}(x)$ являются нечеткими, при математической формулировке этих ограничений использован нечеткий подход и в дальнейшем при решении этой задачи эффективно применяются идеи многокритериальной оценки альтернатив, компромиссных схем, модифицированные для работы в нечеткой среде.

Приведем классификацию задач многокритериальной оптимизации применительно к специфике нефтеперерабатывающих, нефтехимических и химических производств. Можно выделить следующие виды рассматриваемых задач:

- с четкими целевыми функциями (критериями), четкими управляющими переменными, четкими ограничениями на управления, четкими функциональными ограничениями (на целевые функции);

- с четкими целевыми функциями, четкими управляющими переменными, четкими ограничениями на управления, нечеткими функциональными ограничениями;

- с четкими целевыми функциями, четкими управляющими переменными, нечеткими ограничениями на управления, нечеткими функциональными ограничениями.

Возможны другие виды задач оптимизации, которые получаются в других комбинациях. Нами в данной работе исследуется и решается задача многокритериальной оптимизации при управлении XTC производства бензола второго вида, т. е. с четкими целевыми функциями, четкими управляющими переменными, четкими ограничениями на управления и нечеткими функциональными ограничениями;

Формализация многокритериальной задачи оптимизации при управлении режимами работы ХTC в нечеткой информационной среде.

Пусть $f(x)=f_{1}(x), \ldots f_{m}(x)$ вектор частных критериев управления, оценивающий режимы работы, т. е. качество работы ХTC. Значения этих критериев зависят от $x=\left(x_{1}, \ldots, x_{n}\right)$, т. е. от вектора режимных параметров $[14,15,25-28]$. Предположим, что эту зависимость описывают математические модели, разрабатываемые на основе вышеприведенного метода. Нечеткие ограничения на 
качественные показатели объекта могут быть описаны как $\varphi_{q}(x) \tilde{\geq} b_{q}, q=\overline{1, L}$.

Требуется выбрать такие значения режимных параметров $x=\left(x_{1}, \ldots, x_{n}\right)$, которые обеспечивают наилучшие значения вектора критериев управления $f(x)=f_{1}(x), \ldots f_{m}(x)$, при выполнении заданных нечетких ограничений. При выборке режимов работы необходимо учитывать предпочтения ЛПР.

Мателатическая постановка задачи. Формализованную задачу управления, в условиях многокритериальности и нечеткости, можно записать в виде следующей постановки:

$$
\begin{gathered}
\max _{x \in X} f_{i}(x), i=\overline{1, m} ; \\
X=\left\{x \in \Omega, \varphi_{q}(x) \tilde{>} b_{q}, q=\overline{1, L}\right\},
\end{gathered}
$$

где $f_{i}(x), i=\overline{1, m}$ - частные критерии управления, значения которых вычисляются по моделям $[11,29,30] ; \varphi_{q}(x), q=\overline{1, L}-$ функции ограничений, определяющие допустимую область $\Omega$ многокритериальной задачи $(6),(7) ; b_{q}, q=\overline{1, L}-$ заданные числа, которые могут быть нечеткими.

Решением данной задачи многокритериальной оптимизации режимов работы ХТС является значения режимных параметров $\mathbf{x}^{*}=\left(x_{1}^{*}, \ldots, x_{n}^{*}\right)$, обеспечивающие наилучшие значения критериев $f_{i}(x)$, $i=\overline{1, m}$, которые удовлетворяют ЛПР.

В известных работах постановки таких задач в нечеткой среде и методы их решения в основном рассматриваются применительно к однокритериальным случаям, нет гибкости в учете предпочтений ЛПР. Кроме того, как правило, исходная нечеткая задача на этапе постановки с применением множеств уровня $\alpha$ заменяется системой эквивалентных детерминированных задач, что приведет к потере основной части собранной нечеткой информации $[14,16,21,31]$.

Часто при многокритериальной оптимизации при управлении ХTC в реальных условиях для ЛПР более привычной и основной исходной информацией является нечеткая информация в виде нечеткого высказывания и суждения специалистов. При этом преобразовать исходную нечеткую информацию в четкую информацию часто не удается или оказывается нецелесообразным. В этих условиях для эффективного решения рассматриваемой задачи необходимо разработать и применить эвристические методы, основанные на привлечение ЛПР, приспособленные к человеческим процедурам принятия решений по управлению. То есть задача многокритериальной оптимизации ставится и решается в нечеткой информационной среде, не преобразуя нечетких задач к системе детерминированных задач. С этой целью в данной работе для решения задачи многокритериальной оптимизации при управлении режимами работы XTC в нечеткой среде предложен эвристический подход, который применяет новые и комбинацию компромиссных схем, адаптированных к применению нечеткой информации.
Постановку задачи многокритериальной оптимизации (6), (7) перепишем применительно к управлению режимами работы ХТС на основе методологии ТНМ.

Введем следующие обозначения: $\mu_{0}(x)=\left(\mu_{0}^{1}(x), \ldots, \mu_{0}^{m}(x)\right)-$ нормализованные критерии $f_{i}(x), i=1, m$, определяющие качества работы ХTC, принимающие значения в интервале $[0,1]$; $\mu_{q}(x), q=1, L-$ функции принадлежности выполнения нечетких ограничений $\varphi_{q}(x) \tilde{\geq} b_{q}, q=\overline{1, L}$; $\gamma=\left(\gamma_{1}, \ldots, \gamma_{m}\right), \beta=\left(\beta_{1}, \ldots, \beta_{L}\right)-$ соответственно, весовые векторы, задаваемые ЛПР и определяющие взаимную важность критериев и ограничений.

С целью адаптации различных компромиссных схем для обеспечения работы при нечеткой информации можно сформулировать различные задачи многокритериальной оптимизации при управлении режимами работы ХTC в виде задач многокритериальной нечеткой оптимизации (нечеткого математического программирования) и разработать методы их решения [15, 30, 32].

Часто на практике при решении рассмотренных задач достаточно выполнения некоторых принципов оптимальности с определенной уступкой. Предлагаем использовать модифицированные для работы в нечеткой среде приниипы квазилаксилина (для критериев) и идеальной точки (для ограничений).

Тогда постановка многокритериальной задачи оптимизации при управлении режимами работы ХTC в нечеткой среде имеет вид:

$$
\max _{x \in X} \mu_{0}^{1}(x),
$$

$X=\left\{\begin{array}{l}x: \arg \max _{x \in \Omega} \min _{i \in I_{0}}\left(\gamma_{i} \mu_{0}^{i}(x)-\Delta_{i}\right) \wedge \arg \left(\mu_{q}(x) \geq\right. \\ \left.\geq \min \left\|\mu(x)-\mu^{u}\right\|_{D}\right), I_{0}=\{2, \ldots, m\}, q=\overline{1, L}\end{array}\right\}$.

В (9) $\left\|\mu(x)-\mu^{u}\right\|_{D}-$ метрика, оценивающая расстояние от текущего решения $\mu(x)$ до идеального решения $\mu^{u} ; \mu^{u}=\left(\max \mu_{1}(x), \ldots, \max \mu_{L}(x)\right)-$ координата идеальной точки (решения), если функции принадлежности нормальные, то $\mu^{u}=(1, \ldots, 1) ; \Omega$ - исходное множество определения переменных $x, I_{0}-$ множество индексов критериев, перенесенных в ограничения.

В постановке (8), (9) при управлении режимами работы XTC решается задача максимизации самого важного критерия с приоритетом 1 , остальные частные критерии вводятся в состав ограничений на основе принципа квазимаксимина с учетом введенных ЛПР уступок $\Delta_{i}, i=\overline{2, m}$, а степени выполнения нечетких ограничений проверяются на основе идеи принципа идеальной точки.

Рассмотрим другую комбинацию использования принципов оптимальности: максилина (для критериев) и Парето оптимальности (для ограничений), при постановке и решении задачи многокритериальной оптимизации при управлении режимами работы ХТС. При этом исходную задачу можно записать в следующей постановке:

$$
\max _{x \in X} \mu_{0}^{1}(x)
$$




$$
X=\left\{\begin{array}{l}
\arg \max _{x \in \Omega} \min _{i \in I_{0}}\left(\gamma_{i} \mu_{0}^{i}(x)\right) \wedge \\
\wedge \arg \max _{x \in \Omega} \sum_{q=1}^{L} \beta_{q} \mu_{q}(x) \wedge \\
\wedge \sum_{q=1}^{L} \beta_{q}=1 \wedge \beta_{q} \geq 0, I_{0}=\{2, \ldots m\}, q=\overline{1, L}
\end{array}\right\} .
$$

В задаче (10), (11) максимизируется главный критерий с приоритетом 1 , остальные критерии вводятся в состав ограничений по принципу максимина (MМ), а нечеткие ограничения учитывается на основе принципа Парето оптимальности (ПО). Предлагается следующий эвристический алгоритм для решения задачи (10), (11).

Алг г р и т м $M M+\Pi O:$

1. В диалоговом режиме с ЛПР определяются значения весовых коэффициентов для частных критериев

$$
\mu_{0}^{i}(x), i=\overline{1, m}, \gamma=\left(\gamma_{1}, \ldots, \gamma_{m}\right), \sum_{i=1}^{m} \gamma_{j}=1, \gamma_{i} \geq 0 .
$$

2. В диалоге с ЛПР определяются значения весовых коэффициентов для ограничений $\mu_{q}(x)$,

$$
q=\overline{1, L}: \beta=\left(\beta_{1}, \ldots, \beta_{L}\right), \sum_{q=1}^{L} \beta_{q}=1, \beta_{q} \geq 0, q=\overline{1, L} \text {. }
$$

3. Задается $p_{q}(x), q=\overline{1, L}-$ число шагов по каждой $q$-й координате.

4. С целью изменения координат вектора $\beta_{q}$ по выражению $h_{q}=\frac{1}{p_{q}}, q=\overline{1, L}$ определяются величины шагов.

5. Варьируя координаты с шагом $h_{q}$ в интервале $[0,1]$, определяется набор весовых векторов $\beta^{1}, \beta^{2}, \ldots, \beta^{N}, N=\left(p_{1}+1\right) \times\left(p_{2}+1\right) \times \ldots \times\left(p_{L}+1\right)$.

6. С привлечением ЛПР, экспертов выбирается терм-множество и строятся функции принадлежности выполнения для нечетких ограничений $\mu_{q}(x), q=\overline{1, L}$.

7. На основе модели XTC решается задача максимизации главного (первого) критерия $\max _{x \in X} \mu_{0}^{1}(x)$ (10) на множестве $X$, которое определяется по выражению (11). Определяются текущие решения: значения режимных параметров $x(\gamma, \beta)$; значения критериев $\mu_{0}^{1}(x(\gamma, \beta)), \ldots, \mu_{0}^{m}(x(\gamma, \beta))$ и степени выполнения нечетких ограничений $\mu_{1}(x(\gamma, \beta)), \ldots, \mu_{L}(x(\gamma, \beta))$.

8. ЛПР анализирует текущие решения. Если текущие решения не удовлетворяют ЛПР, то им корректируются значения весовых коэффициентов $\gamma_{1}, \ldots, \gamma_{m} \gamma$ и/или $\beta_{1}, \ldots, \beta_{L} \beta$, и выполняется переход обратно к пункту 3 . В противном случае, перейти к следующему пункту 9.

9. Прекращается поиск решений, и выводятся оптимальные, т. е. выбранные ЛПР, окончательные результаты, которые обеспечивают оптимальные режимы при управлении XTC: оптимальные зна- чения режимных (управляющих) параметров $x^{*}(\gamma, \beta)$, обеспечивающие лучшие значения частных критериев $\mu_{0}^{1}\left(x^{*}(\gamma, \beta)\right), \ldots, \mu_{0}^{m}\left(x^{*}(\gamma, \beta)\right)$ и максимальные степени выполнения нечетких ограничений $\mu_{1}\left(x^{*}(\gamma, \beta)\right), \ldots, \mu_{L}\left(x^{*}(\gamma, \beta)\right)$.

Таким образом, получены различные постановки задачи многокритериальной оптимизации при управлении режимами работы XTC в нечеткой информационной среде. На основе различных принципов оптимальности (максимина и Парето оптимальности) и методов THМ предложен новый эвристический алгоритм решения ММ+ПО.

\section{Применение и обсуждение результатов}

В качестве примера реализации предложенного нечеткого подхода к решению задачи многокритериальной оптимизации при управлении режимами работы XTC рассмотрим постановки и решения задачи управления режимами работы ХTС производства бензола Атырауского НПЗ. Используя вышеприведенные результаты исследования и на основе модификации методов максимина и принципа Парето оптимальности, задачу многокритериальной оптимизации режимов работы XTC производства бензола можно формализовать и сформулировать следующим образом:

Введем следующие обозначения: $\mu_{0}(x)=\left(\mu_{0}^{1}(x)\right.$, $\left.\mu_{0}^{2}(x), \mu_{0}^{3}(x)\right)$ - нормализованный критерий (в интервале $[0,1])$, оценивающий выход продукции ХTC производства бензола $\left(\mu_{0}^{1}(x)\right.$ - выход бензола, $\mu_{0}^{2}(x)$ - выход рафината, $\mu_{0}^{3}(x)$ - выход тяжелой ароматики); $\mu_{1}(x), \mu_{2}(x)$ - ФП выполнения нечетких ограничений $\varphi_{q}(x) \tilde{>} b_{q}, q=1,2 ; \gamma=\left(\gamma_{1}, \gamma_{2}, \gamma_{3}\right)$ и $\beta=\left(\beta_{1}, \beta_{2}\right)$ - весовые векторы, отражающие взаимную важность критериев и ограничений.

Тогда математическую постановку задачи многокритериальной оптимизации при управлении ХTC производства бензола на основе задачи (10), (11) можно записать в следующем виде:

$$
\max _{x \in X} \mu_{0}^{1}(x),
$$

$$
X=\left\{\begin{array}{l}
\arg \max _{x \in \Omega} \min _{i \in I_{0}}\left(\gamma_{i} \mu_{0 R}^{i}(x)\right) \wedge \\
\wedge \arg \max _{x \in \Omega} \sum_{q=1}^{2} \beta_{q} \mu_{q}(x) \wedge \\
\wedge \sum_{q=1}^{2} \beta_{q}=1 \wedge \beta_{q} \geq 0, I_{0}=\{2,3\}, q=\overline{1,2}
\end{array}\right\} .
$$

В постановке (12)-(13): ^ - логический «и», который означает, что все связываемые им утверждения являются истинными: $\mu_{0 R}^{i}$ - введенные ЛПР ограничения для критериев $\mu_{0}^{i}(x), i=2,3$. Варьируя значения весовых коэффициентов локальных критериев $\gamma_{1}, \gamma_{2}, \gamma_{3}$ и ограничений $\beta_{1}, \beta_{2}$, получаем семейство решений задачи (12), (13), среди которых ЛПР выбирает оптимальные значения режимных параметров $x^{*}(\gamma, \beta)$, обеспечивающие максимальные значения критериев, не нарушая ограничений. 
Для решения поставленной задачи многокритериальной оптимизации при управлении режимами работы ХТС производства бензола (12), (13) используем комбинацию модифицированных методов максилина (ММ) и Парето оптилальности (ПО).

Приведем конкретизацию алгоритма $M M+\Pi O$ для решения задачи (12), (13).

1. В диалоге с ЛПР определены и введены значения весовых коэффициентов частных критериев $\mu_{0}^{i}(x)$,

$$
i=\overline{1,3}, \sum_{i=1}^{3} \gamma_{i}=1, \gamma_{i} \geq 0, i=1,2,3: \gamma=(0,7 ; 0,2 ; 0,1) \text {. }
$$

2. В диалоге с ЛПР экспертами определены значения весовых коэффициентов для ограничений $\mu_{q}(x), q=\overline{1,3}, \sum_{q=1}^{2} \beta_{q}=1, \beta_{q} \geq 0, q=1,2: \beta=(0,7 ; 0,3)$.

3. ЛПР заданы $p_{q}, q=1,2$ - количество шагов по координатам: $p_{1}=5 ; p_{2}=2$.

4. Вычислены $h_{q}=\frac{1}{p_{q}}, q=\overline{1,2}-$ величины шагов для изменения координат весового вектора $\beta_{q}$ :

$$
h_{1}=\frac{1}{p_{1}}=\frac{1}{5}=0,2 ; h_{2}=\frac{1}{p_{2}}=\frac{1}{2}=0,5 .
$$

5. Определены весовые векторы $\beta^{1}, \beta^{2}, \ldots, \beta^{N}$, $N=(5+1) \times(2+1)=18$ варьированием координат на отрезках $[0,1]$ с шагом $h_{q}, q=1,2$.

6. Определено терм-множество и построены ФП выполнения ограничений $\mu_{q}(x), q=1,2$. Задача описывается двумя нечеткими ограничениями: «среднее октановое число бензола $\leq 102$ » и «доля серы в составе бензола $\leq 0,00005 \%$. Для описания этих нечетких ограничений определено следующее терм-множество: $T(X, Y)=\{$ низкое, ниже среднего, среднее, выше среднего, высокое \}. С применением приведенного терммножества $T(X, Y)$ на основе формулы (1) построены ФП, описывающие степени выполнения нечетких ограничений:

$$
\begin{gathered}
\mu_{1}^{1}(x)=\exp \left(\left(0.5 \mid y_{4}-97\right) 0.60 \mid\right) ; \\
\mu_{1}^{2}(x)=\exp \left(\left(0.5 \mid y_{4}-100\right) 0.55 \mid\right) ; \\
\mu_{1}^{3}(x)=\exp \left(\left(0.5 \mid y_{4}-102\right) 0.50 \mid\right) ; \\
\mu_{1}^{4}(x)=\exp \left(\left(0.5 \mid y_{4}-104\right) 0.55 \mid\right) ; \\
\mu_{1}^{5}(x)=\exp \left(\left(0.5 \mid y_{4}-107\right) 0.60 \mid\right) ; \\
\mu_{2}^{1}(x)=\exp \left(\left(0.3 \mid y_{5}-0.000005\right) 0.15 \mid\right) ; \\
\mu_{2}^{2}(x)=\exp \left(\left(0.3 \mid y_{5}-0.000020\right) 0.12 \mid\right) ; \\
\mu_{2}^{3}(x)=\exp \left(\left(0.3 \mid y_{5}-0.000050\right) 0.10 \mid\right) ; \\
\mu_{2}^{4}(x)=\exp \left(\left(0.3 \mid y_{5}-0.000080\right) 0.12 \mid\right) ; \\
\mu_{2}^{5}(x)=\exp \left(\left(0.3 \mid y_{5}-0.000100\right) 0.15 \mid\right),
\end{gathered}
$$

где $\mu_{1}^{p}(x), \mu_{2}^{p}(x), p=\overline{1,5}$ - ФП, описывающие степени выполнения нечетких ограничений для каждого кванта $p$ на среднее октановое число бензола $\left(\mu_{1}^{p}(x)\right)$ и доли серы в составе бензола $\left(\mu_{2}^{p}(x)\right), y_{4}$ и $y_{5}$ - числовые значения нечетких по- казателей качества бензола, полученные на основе множества уровня $\alpha$, остальные коэффициенты рассмотрены при описании формулы (1).

7. На основе вышеприведенных моделей (3)-(5) и нечетких ограничений, описывающих зависимости частных критериев от режимных параметров: $x_{1}, x_{2}, x_{3}, x_{4}, x_{5}$, решена задача $\max _{x \in X} \mu_{0}^{1}(x)$ (12) на множестве $X$ (13). Критерии максимизированы на множестве $X$ с учетом коэффициентов важности, которые определены в пункте 1. Определены текущие решения: значения режимных параметров $x(\gamma, \beta)$ и соответствующие им значения частных критериев $\mu_{0}^{1}\left(x^{*}(\gamma, \beta)\right)$, $\mu_{0}^{2}\left(x^{*}(\gamma, \beta)\right), \mu_{0}^{3}\left(x^{*}(\gamma, \beta)\right)$, и степени выполнения нечетких ограничений $\mu_{1}\left(x^{*}(\gamma, \beta)\right), \mu_{2}\left(x^{*}(\gamma, \beta)\right)$. Здесь можно применить наиболее подходящий метод решения поставленной задачи, в нашем случае использован модифицированный для работы в нечеткой среде метод штрафных функции.

8. Полученные решения предъявлены ЛПР. Если текущие результаты не удовлетворяют ЛПР, то он производит корректировку значения $\gamma$ и (или) $\beta$, и осуществляется возврат к пункту 2 . В случае удовлетворения ЛПР осуществляется переход к пункту 9. В нашем случае ЛПР удовлетворяющие его решения выбрал после 5-го цикла, и эти результаты занесены в таблицу.

9. Остановка поиска решения, вывод удовлетворяющих ЛПР окончательных решений, обеспечивающих оптимальный режим ХТС производства бензола: оптимальные значения режимных параметров $x_{1}^{*}(\gamma, \beta), x_{2}^{*}(\gamma, \beta), x_{3}^{*}(\gamma, \beta), x_{4}^{*}(\gamma, \beta)$, $x_{5}^{*}(\gamma, \beta)$; максимальные значения частных критериев $\mu_{0}^{1}\left(x^{*}(\gamma, \beta)\right), \mu_{0}^{2}\left(x^{*}(\gamma, \beta)\right), \mu_{0}^{3}\left(x^{*}(\gamma, \beta)\right)$ и максимальные степени выполнения нечетких ограничений $\mu_{1}\left(x^{*}(\gamma, \beta)\right), \mu_{2}\left(x^{*}(\gamma, \beta)\right)$ (таблица).

Обсуждение результатов. В результате анализа данных, приведенных в таблице, можно сделать следующие выводы:

1. Предложенный эвристический алгоритл $M M+\Pi О$ позволяет решить исходную задачу с нечеткими ограничениями без предварительного преобразования их в детеминировыанный вариант, по сравнению с детерминированным методом, по некоторым показателям обеспечивает лучшие результаты.

2. При решении многокритериальной задачи в нечеткой постановке адекватность решения улучшается, так как дополнительно используется нечеткая информация (знание, опыт ЛПР и экспертов), позволяющая аддекватно описать реальную ситуацию без идеализации.

3. Алгоритм $M M+П O$ позволяет определить функцию принадлежности, т. е. степень выполнения нечетких ограничений, обеспечивает решение задачи с нечеткими ограничениями, что часто возникают в производственных условиях.

4. В процессе решение задачи с помощью ЛПР можно определить компромиссное решение между количеством и качеством продукции. 
таблица. Сравнение результатов оптимизации режимов работы ХTC по детерминированному методу, по предложенному алгоритму и реальных значений

Table. $\quad$ Comparison of Chemical-technological system work modes' optimization results by deterministic method, proposed algorithm and actual values

\begin{tabular}{|c|c|c|c|}
\hline $\begin{array}{l}\text { Значения критериев управления и ограничений } \\
\text { Values of control criteria and constraints }\end{array}$ & $\begin{array}{c}\text { Детерминирован- } \\
\text { ный метод [33] } \\
\text { Deterministic } \\
\text { method [33]) }\end{array}$ & \begin{tabular}{c|} 
Предложенный \\
алгоритм \\
Proposed \\
algorithm
\end{tabular} & $\begin{array}{c}\text { Реальные } \\
\text { значения } \\
\text { Actual } \\
\text { values }\end{array}$ \\
\hline $\begin{array}{l}\text { Выход бензола с бензольной колонны, кг - критерий } y_{1} \\
\text { Output of benzene from the benzene column, } \mathrm{kg} \text { - criterion } y_{1}\end{array}$ & $1,33 \cdot 10^{8}$ & $1,341 \cdot 10^{9}$ & $1,30 \cdot 10^{8}$ \\
\hline $\begin{array}{l}\text { Выход рафината с бензольной колонны, кг - критерий } y_{2} \\
\text { Output of the raffinate from the benzene column, } \mathrm{kg} \text { - criterion } y_{2}\end{array}$ & $8,2 \cdot 10^{7}$ & $8,24 \cdot 10^{8}$ & $8,0 \cdot 10^{7}$ \\
\hline $\begin{array}{l}\text { Выход тяжелой ароматики с ректификационной колонны, кг - критерий } y_{3} \\
\text { Output of heavy aromatics from the distillation column, } \mathrm{kg}-\text { criterion } y_{3}\end{array}$ & $4,50 \cdot 10^{8}$ & $4,50 \cdot 10^{8}$ & $4,50 \cdot 10^{8}$ \\
\hline $\begin{array}{l}\text { ФП выполнения нечеткого ограничения. } \\
\text { Среднее октановое число бензола должно быть не менее } \tilde{\geq} 102-\mu_{1}\left(x^{*}(\beta)\right) \\
\text { Membership function fulfillment of a fuzzy constraint. } \\
\text { Average octane number of benzole should be at least } \Sigma 102-\mu_{1}\left(x^{*}(\beta)\right)\end{array}$ & - & 1,0 & - \\
\hline $\begin{array}{l}\text { ФП выполнения нечеткого ограничения. } \\
\text { Содержание серы в составе бензола должно быть не более } \tilde{\leq} 0,00005 \%-\mu_{2}\left(x^{*}(\beta)\right) \\
\text { Membership function fulfillment of a fuzzy constraint. } \\
\text { Sulfur content of benzole should be no more than } \tilde{\leq} 0,00005 \%-\mu_{2}\left(x^{*}(\beta)\right)\end{array}$ & - & 1,0 & - \\
\hline $\begin{array}{l}\text { Оптимальные значения режимных параметров/Optimal values of the operating parameters: } \\
x_{1}^{*} \text { - сырье, вход реформата, кг; raw materials, the input of the reformat, kg; } \\
x_{2}{ }^{*} \text { - температура в бензольной колонне, К; temperature in the benzole column, K; } \\
x_{3}{ }^{*} \text { - давление в бензольной колонне, кПа; pressure in the benzole column, } \mathrm{kPa} ; \\
x_{4}{ }^{*} \text { - доля серы в составе сырья, \%; share of sulphur in the composition of raw materials, \%; } \\
x_{5}^{*} \text { - доля аром. УВ в составе сырья, \%; share of aromatic hydrocarbons in the composition of } \\
\text { raw materials, \% }\end{array}$ & $\begin{array}{l}7,46 \cdot 10^{8} \\
423,5 \\
3628 \\
0,00005 \\
50\end{array}$ & $\begin{array}{l}7,46 \cdot 10^{8} \\
418 \\
3432 \\
0,00005 \\
50\end{array}$ & $\begin{array}{l}7,46 \cdot 10^{8} \\
423,5 \\
3628 \\
0,00005 \\
\\
50\end{array}$ \\
\hline
\end{tabular}

Примечание: В детерминированном подходе и на практике степени выполнения нечетких ограничений не определяются, поэтому в соответствующие графы таблицы занесены (-). Реальные значения параметров в таблице соответствуют значениям технологических параметров одного из режимов установки производства бензола Атырауского НПЗ.

Note: In the deterministic approach and in practice, the degree of implementation of fuzzy constraints is not defined, therefore (-) iszzz entered in the corresponding columns of the table. The actual values of the parameters in the table correspond to the values of the technological parameters of one of the modes of the installation of benzene production of the Atyrau refinery.

Таким образом, чтобы улучшить качество продукции придется уменьшить объем её выпуска. Поэтому постановка задачи максимизации объема выхода бензола с одновременным улучшением его качества является некорректной. В этом случае может быть два варианта корректной постановки задачи:

1) максимизация выхода продукции с обесечением показателей качества не менее чем заданные значения, т. е введением и учетом ограничения на качество;

2) максимальное улучшение качества продукции с обесечением заданного объема на выход продукции, т. е. введением и учетом ограничения на объем продукции.

Приведенные в таблице результаты показывают эффективность предложенного эвристического алгорима решения задачи управления режимами работы XTC в нечеткой постановке, так как по сравнению с результатами известных методов [33] по всем показателям он показывает не худшие результаты, а по выходу бензола и рафината результаты улучшены.

Кроме того, алгоритм $\mathrm{MM}+\Pi 0$ позволяет учитывать нечеткие ограничения, определяет степени выполнения нечетких ограничений. Как видно из таблицы, обеспечено полное выполнение нечетких ограничений, т. е. их функции принадлежности равны $1: \mu_{1}\left(x^{*}(\gamma, \beta)\right)=1 ; \mu_{2}\left(x^{*}(\gamma, \beta)\right)=1$.

\section{Заключение}

Разработан метод построения математических моделей ХTC в условиях неопределенности и нечеткости исходной информации на основе информации различного характера. Впервые получены постановки задач управления режимами работы XTC при нечеткой информации в виде задачи нечеткого математического программирования. На основе модификации методов максимина и Парето оптимальности для работы в нечеткой информационной среде разработан эвристический алгоритл ММ+ПО решения полученной задачи. Новизна и оригинальность результатов заключается в том, что задачи многокритериальной оптимизации при управлении ХТС ставятся и решаются в нечеткой информационной среде без предварительного преобразования их к детерминированным эквивалентным задачам. Это обеспечивает правильность и эффективность управления за счет повышения адекватности описания производственных задач при нечеткой информации.

Предложенные подходы реализованы при построении математических моделей и решении задачи многокритериальной оптимизации при упра- 
влении режимами работы ХТС производства бензола при нечеткой информации. Результаты исследования расширяют круг решаемых практических

\section{СПИСОК ЛИТЕРАТУРЫ}

1. Джарратино Д. Экспертные системы: принципы разработки и программирование. 4-е изд. - М.: 000 «И.Д. Вильямс», 2007. - $1152 \mathrm{c}$.

2. Gronostajski Z. The expert system supporting the assessment of the durability of forging tools // International journal of advanced manufacturing technology. - 2016. - V. 82. - № 9. P. $1973-1991$.

3. Sabzi H.Z. Developing an intelligent expert system for streamflow prediction, integrated in a dynamic decision support system for managing multiple reservoirs: a case study // Expert systems with applications. - 2017. - V. 82. - № 3. - P. 145-163.

4. Expert system for polyester exhaust dyeing / J.J. Lin, J. Lee, K.L. Cheng, F.B. Lin // AATCC Review. - 2001. - V. 1. - № 1. P. 41-44.

5. Kahraman C. Fuzzy Multi-Criteria Decision Making // Theories and Applications with Recent Developments. - New York: Springer, 2008. - P. 592-608.

6. Dubois D. The role of fuzzy sets indecision sciences: old techniques and new directions // Fuzzy Sets and Systems. - 2011. V. $184 .-$ P. $3-17$.

7. Сулейменов Б.А. Интеллектуальные и гибридные системы управления технологическими процессами. - Алматы: Изд-во «Пикула и К», 2009. - 304 с.

8. Оразбаев Б.Б. Теория и практика методов нечетких множеств. - Алматы: Изд-во «Бастау», 2014. - 455 с.

9. Рыжов А.П. Теория нечетких множеств и ее приложений. М.: Изд-во МГУ, 2017. -115 с.

10. Grossmann I.E. Challenges in the Application of Mathematical Programming in the Enterprise-wide 0ptimization of Process Industries // Theoretical Foundations of Chemical Engineering. 2014. - V. 48. - № 5. - P. 500-518.

11. Orazbayev B.B., Orazbayeva K.N., Utenova B.E. Development of Mathematical Models and Modeling of Chemical Engineering Systems under Uncertainty // Theoretical Foundations of Chemical Engineering. - 2014. - V. 48. - № 4. - P. 138-149.

12. Волин Ю.М., Островский Г.М. Многокритериальная оптимизация технологических процессов в условиях неопределенности // Автоматика и телемеханика. - 2007. - Т. 53. - № 3. C. $165-178$.

13. Pavlov S.Yu., Kulov N.N., Kerimov R.M. Improvement of Chemical Engineering Processes Using Systems Analysis // Theoretical Foundations of Chemical Engineering. -2016. - V. 53. № 2. - P. 117-133.

14. Зайченко Ю.П. Исследование операций: нечеткая оптимизация. - Киев: Изд-во Выща школа, 1991. - 357 с.

15. Multicriteria optimisation problems for chemical engineering systems and algorithms for their solution based on fuzzy mathematical methods / B.B. Orazbayev, K.N. Orazbayeva, L.T., Kurmangaziyeva V.E. Makhatova // EXCLI Journal. - 2015. - V. 14. P. 984-998.

16. Biegler L.T., Lang Y.D, Lin W.J. Multi-scale Optimization for Process Systems Engineering // Computers and Chemical Engineering. - 2016. - № 10. - P. 17-35.

17. Секретарев Ю.А., Мятеж Т.В., Мошкин Б.Н. Математическая модель управления функционированием генерирующей компании в современных условиях // Известия Томского политехнического университета. Инжиниринг георесурсов. - 2018. T. 329. - № 2. - С. 146-158.

18. Валиахметов Р.И. и др. Применение эвристических алгоритмов в анализе данных для решения задачи диагностирования электроцентробежных насосных установок // Известия Том- задач, позволяют моделировать и управлять режимами работы сложных XTC с учетом их многокритериальности и нечеткости исходной информации.

ского политехнического университета. Инжиниринг георесурсов. -2018. - Т. 329. - № 2. - С.159-167.

19. Control of Fuzzy Technological Objects Based on Mathematical Model / B.B. Orazbayev, Y.A. Ospanov, K.N. Orazbayeva, T. Gancarzyk, A. Shaikhanova // $16^{\text {th }}$ International Conference on Control, Automation and Systems (ICCAS 2016). - Gyengju, Korea, 18-19 October 2016. - P. 1487-1493.

20. Технологический регламент технологического комплекса по производству бензола. - Атырау: Атырауский нефтеперерабатывающий завод, 2017. - 387 с.

21. A Blended Risk Index Modeling and Visualization Based on Hierarchical Fuzzy Logic for Water Supply Pipelines Assessment and Management / M. Fayaz, S. Ahmad, I. Ullah, D. Kim // Processes. - 2018. - V. 6. - № 5. - P. 102-112.

22. Дмитриевский Б.С., Затонский А.В., Тугашова Л.Г. Задача управления процессом ректификации нефти и метод ее решения // Известия Томского политехнического университета. Инжиниринг георесурсов. - 2018. - Т. 329. - № 2. - С. 136-145.

23. Леоненков А. Нечеткое моделирование в среде MATLAB и fuzzуTЕСН. - СПб.: Изд-во «Петербург», 2003. - 787 с.

24. Optimization of Chemical Process Design with Chance Constraints by an Iterative Partitioning Approach / G.M. Ostrovsky, N.N. Ziyatdinov, T.V. Lapteva, A. Silvestrova / Journal of Industrial and Engineering Chemistry. - 2015. - V. 54. - № 13. - P. 3412-3437.

25. Optimization-based design of crude oil distillation units using surrogate column models and a support vector machine / D. Ibrahim, M. Jobson, J. Li, G. Guillen-Gosalbez // Chemical engineering research \& design. - 2018. - V. 134. - P. 212-225.

26. Multi-criteria design of shale-gas-water supply chains and production systems towards optimal life cycle economics and greenhouse gas emissions under uncertainty / Y. Chen, L. He, J. Li, S. Zhang // Computers \& chemical engineering. - 2018. V. $109 .-$ P. $216-235$.

27. Harinath E., Biegler L.T., Dumont G.A. Predictive optimal control for thermo-mechanical pulping processes with multi-stage low consistency refining // Journal of Process Control. - 2013. V.47. - № 23. - P. 1001-1015.

28. Novak Z., Kravanja Z. Mixed-integer nonlinear programming problem process synthesis under uncertainty by reduced dimensional stochastic optimization // Industrial \& engineering chemistry research. - 1999. - V. 38. - № 7. - P. 2680-2698.

29. Мешалкин В.П., Гимаров В.А., Зайцевский И.В. Локальные математические модели для управления промышленным предприятием. - М.: Изд-во физико-математической литературы, 2003. -456 c.

30. Оразбаев Б.Б. Методы моделирования и принятия решений для управления производством в нечеткой среде. - Астана: Изд-во Евразийский национальный университет имени. Л.Н. Гумилева, 2016. - 398 с.

31. State-of-the-art review of optimization methods for short-term scheduling of batch processes / C.A. Méndez, J., Cerdá I.E. Grossmann, I. Harjunkoski, M. Fahl // Computers and Chemical Engineering. - 2006. - № 30. - P. 913-928.

32. Grossmann I.E. Challenges in the Application of Mathematical Programming in the Enterprise-wide Optimization of Process Industries // Теоретические основы химической технологии. 2014. - T. 48. - № 5. - C. 500-517.

33. Шумский В.М., Зырянова Л.А. Инженерные задачи в нефтепереработке и нефтехимии. - М.: Изд-во «Химия», 1981. - 457 с.

Поступила 15.11.2018 2. 


\section{Информация об авторах}

Оразбаев Б.Б., доктор технических наук, профессор кафедры Системного анализа и управления Евразийского национального университета им. Л.Н. Гумилева.

Ocnaнов E.A., докторант кафедры Системного анализа и управления Евразийского национального университета им. Л.Н. Гумилева.

Оразбаева $\boldsymbol{K} . \boldsymbol{H}$. доктор технических наук, профессор кафедры Менеджмента и маркетинга Казахского университета экономики, финансов и международной торговли.

Серимбетов Б.А., кандидат технических наук, доцент, заведующий кафедры информатики и прикладной экономики Казахского университета экономики, финансов и международной торговли. 


\title{
MULTICRITERIA OPTIMIZATION IN CONTROL OF A CHEMICAL-TECHNOLOGICAL SYSTEM FOR PRODUCTION OF BENZENE WITH FUZZY INFORMATION
}

\author{
Batyr B. Orazbayev', \\ batyr_o@mail.ru \\ Yerbol A. Ospanov', \\ 78oea@mail.ru \\ Kulman N. Orazbayeva², \\ kulman_o@mail.ru \\ Bulat A. Serimbetov ${ }^{2}$, \\ sba_mmc@mail.ru \\ 1 L.N. Gumilev Eurasian National University, \\ 11, Pushkin Street, Astana, 010008, Kazakhstan. \\ ${ }^{2}$ Kazakh University of Economics, Finance and International Trade, \\ 7, Zhubanov Street, Astana, 010005, Kazakhstan.
}

\begin{abstract}
The relevance of the research is caused by the need to solve effectively the problems of controlling the operating modes of chemicaltechnological systems, which are multi-criteria, characterized by the uncertainty and fuzziness of the initial information. Since real chemical-technological systems in production are multi-criteria and often work in the conditions of unclear initial information, formalization and effective solution of such problems in a fuzzy environment is very important and relevant for science and production.

The main aim of the research is to develop a method for constructing mathematical models of chemical-technological systems based on available information of various kinds (theoretical, statistical, expert, fuzzy information); get multicriteria optimization problem statement to control such systems and develop a heuristic approach to its solution; also, to test the results obtained in practice for construction of mathematical models of chemical-technological system for benzene production and the control modes of its operation in a fuzzy environment.

Objects: complex chemical-technological systems with the example of a technological plant for production of benzene, which are described by a vector of criteria and characterized by unclear initial information.

Methods: methods of system analysis and fuzzy sets theories, methods of mathematical modeling and multi-criteria choice, experimental statistical methods for developing mathematical models.

Results. The authors have proposed the method for developing mathematical models of chemical-technological systems based on available information of various types, including initial fuzzy information. The new tasks of control of the chemical technological systems in the conditions of fuzziness of the initial information obtained by modifications of various compromise schemes for working in a fuzzy environment. The tasks of multicriteria optimization in controlling technological system are reduced to multicriteria problems of fuzzy mathematical programming and based on modifying the principles of maximin and Pareto optimality, heuristic algorithm for solving the formulated problem of optimal control is developed. These results are realized in practice in constructing mathematical models of the main aggregates of the chemical-technological system for production of benzene and in setting and solving the task of controlling the operating modes of this system in a fuzzy environment. Analysis and comparison of the obtained results allow drawing a conclusion about the effectiveness of the proposed approach to solving control problems on the basis of models in conditions of indistinct initial information.
\end{abstract}

Key words:

Chemical-technological system, mathematical modeling, fuzzy information, principles of optimality, benzene, heuristic algorithm, tasks control of operating modes.

\section{REFERENCES}

1. Jarratino D. Ekspertnye sistemy: printsipy razrabotki i programmirovanie [Expert systems: principles of development and programming]. Moscow, I.D. Vilyams Publ., 2007. 1152 p.

2. Gronostajski Z. The expert system supporting the assessment of the durability of forging tools. International journal of advanced manufacturing technology, 2016, vol. 82, no. 9, pp. 1973-1991.

3. Sabzi H.Z. Developing an intelligent expert system for streamflow prediction, integrated in a dynamic decision support system for managing multiple reservoirs: a case study. Expert systems with applications, 2017, vol. 82, no. 3, pp. 145-163.

4. Lin J.J., Lee J., Cheng K.L., Lin F.B. Expert system for polyester exhaust dyeing. AATCC Review, 2001, vol. 1, no. 1, pp. 41-44.
5. Kahraman C. Fuzzy Multi-Criteria Decision Making. Theories and Applications with Recent Developments. New York, Springer, 2008. pp. 592-608.

6. Dubois D. The role of fuzzy sets indecision sciences: old techniques and new directions. Fuzzy Sets and Systems, 2011, vol. 184, pp. 3-17.

7. Suleymenov B.A. Intellektualnye i gibridnye sistemy upravleniya tekhnologicheskimi protsessami [Intelligent and hybrid process control systems]. Almaty, Pikula and K Publ., 2009. 304 p.

8. Orazbayev B.B. Teoriya i praktika metodov nechetkikh mnozhestv [Theory and practice of methods of fuzzy sets]. Almaty, Bastau Publ., 2014. $455 \mathrm{p}$.

9. Ryzhov A.P. Teoriya nechetkikh mnozhestv i ee prilozheniy [The theory of fuzzy sets and its applications]. Moscow, MSU Publ., 2017. $115 \mathrm{p}$. 
10. Grossmann I.E. Challenges in the Application of Mathematical Programming in the Enterprise-wide Optimization of Process Industries. Theoretical Foundations of Chemical Engineering, 2014 , vol. 8, no. 5, pp. 500-518.

11. Orazbayev B.B., Orazbayeva K.N., Utenova B.E. Development of Mathematical Models and Modeling of Chemical Engineering Systems under Uncertainty. Theoretical Foundations of Chemical Engineering, 2014, vol. 48, no. 4, pp. 138-149.

12. Volin Yu.M., Ostrovsky G.M. Multicriteria optimization of technological processes in conditions of uncertainty. Automation and Remote Control, 2007, vol. 53, no. 3, pp. 165-178. In Rus.

13. Pavlov S.Yu., Kulov N.N., Kerimov R.M. Improvement of Chemical Engineering Processes Using Systems Analysis. Theoretical Foundations of Chemical Engineering, 2016, vol. 53, no. 2, pp. 117-133.

14. Zaichenko Yu.P. Issledovanie operatsiy: nechetkaya optimizatsiya [Operations research: fuzzy optimization]. Kyiv, Vyshcha shkola Publ., 1991. 357 p.

15. Orazbayev B.B., Orazbayeva K.N., Kurmangaziyeva L.T., Makhatova V.E. Multicriteria optimisation problems for chemical engineering systems and algorithms for their solution based on fuzzy mathematical methods. EXCLI Journal, 2015, vol. 14, pp. 984-998.

16. Biegler L.T., Lang Y.D, Lin W.J. Multi-scale Optimization for Process Systems Engineering. Computers and Chemical Engineering. 2016, no. 10, pp. 17-35.

17. Sekretarev Y.A., Myatezh T.V., Moshkin B.N. Mathematical model for controlling generation company functioning under modern conditions. Bulletin of the Tomsk Polytechnic University. Geo Assets Engineering, 2018, vol. 329, no. 2, pp. 146-158. In Rus.

18. Valiakhmetov R.I. Application of heuristic algorithms in analyzing data to solve the problem of detection of electric centrifugal pumping units. Bulletin of the Tomsk Polytechnic University. Geo Assets Engineering, 2018, vol. 329, no. 2, pp. 159-167.

19. Orazbayev B.B., Ospanov Y.A., Orazbayeva K.N. Gancarzyk T., Shaikhanova A. Control of Fuzzy Technological Objects Based on Mathematical Model. $16^{\text {th }}$ International Conference on Control, Automation and Systems (ICCAS 2016). Gyengju, Korea, October 18-19, 2016. pp. 1487-1493.

20. Tekhnologicheskiy reglament tekhnologicheskogo kompleksa po proizvodstuu benzol [Technological regulations of the technological complex for the production of benzene]. Atyrau, Atyrau Oil production Plant Publ., 2017. 387 p.

21. Fayaz M., Ahmad, S., Ullah I., Kim D. A Blended Risk Index Modeling and Visualization Based on Hierarchical Fuzzy Logic for Water Supply Pipelines Assessment and Management. Processes, 2018, vol. 6, no. 5, pp. 102-112.

22. Dmitrievsky B.S., Zatonskiy A.V., Tugashova L.G. Task of controlling oil rectification and the method of its solution. Bulletin of the Tomsk Polytechnic University. Geo Assets Engineering, 2018, vol. 329, no. 2, pp. 136-145. In Rus.

23. Leonenkov A. Nechetkoe modelirovanie v srede MATLAB i fuzzyTECH [Fuzzy modeling in the environment of MATLAB and fuzzyTECH]. St-Petersburg, Peterburg Publ., 2003. 787 p.

24. Ostrovsky G.M., Ziyatdinov N.N., Lapteva T.V., Silvestrova A. Optimization of Chemical Process Design with Chance Constraints by an Iterative Partitioning Approach. Journal of Industrial and Engineering Chemistry, 2015, vol. 54, no. 13, pp. 3412-3437.

25. Ibrahim D., Jobson M., Li J., Guillen-Gosalbez, G. Optimizationbased design of crude oil distillation units using surrogate column models and a support vector machine. Chemical engineering research \& design, 2018, vol. 134, pp. 212-225.

26. Chen Y., He L., Li J., Zhang S. Multi-criteria design of shale-gaswater supply chains and production systems towards optimal life cycle economics and greenhouse gas emissions under uncertainty. Computers \& chemical engineering, 2018, vol. 109, pp. 216-235.

27. Harinath E., Biegler L.T., Dumont G.A. Predictive optimal control for thermo-mechanical pulping processes with multi-stage low consistency refining. Journal of Process Control, 2013, vol. 47, no. 23, pp. 1001-1015.

28. Novak Z., Kravanja Z. Mixed-integer nonlinear programming problem process synthesis under uncertainty by reduced dimensional stochastic optimization. Industrial \& engineering chemistry research, 1999, vol. 38, no. 7, pp. 2680-2698.

29. Meshalkin V.P, Gimarov V.A, Zaitsevsky I.V. Lokalnye matematicheskie modeli dlya upravleniya promyshlennym predpriyatiyem [Local mathematical models for industrial enterprise control]. Moscow, Izdatelstvo fiziko-matematicheskoy literatury, 2003. $456 \mathrm{p}$.

30. Orazbayev B.B. Metody modelirovaniya i prinyatiya resheniy dlya upravleniya proizvodstvom $v$ nechetkoy srede [Methods of modeling and decision making for production management in a fuzzy environment]. Astana, L.N. Gumilev Eurasian National University Publ., 2016. 398 p.

31. Méndez C.A., Cerdá J., Grossmann I.E., Harjunkoski I., Fahl M. State-of-the-art review of optimization methods for short-term scheduling of batch processes. Computers and Chemical Engineering, 2006, no. 30, pp. 913-928.

32. Grossmann I.E. Challenges in the Application of Mathematical Programming in the Enterprise-wide Optimization of Process Industries. Teoreticheskie osnovy khimicheskoy tekhnologii, 2014, vol. 48 , no. 5, pp. 500-517.

33. Shumsky V.M., Zyryanova L.A. Inzhenernye zadachi v neftepererabotke i neftekhimii [Engineering tasks in oil refining and petrochemistry]. Moscow, Khimiya Publ., 1981. 457 p.

Received: 15 November 2018.

\section{Information about the authors}

Batyr B. Orazbayev, Dr. Sc, professor, L.N. Gumilev Eurasian National University.

Yerbol A. Ospanov, postgraduate student, L.N. Gumilev Eurasian National University.

Kulman N. Orazbayeva, Dr. Sc, professor, Kazakh University of Economics, Finance and International Trade.

Bulat A. Serimbetov, Cand. Sc., associate professor, Kazakh University of Economics, Finance and International Trade. 